\title{
Exhibitive Nano-to-Micron Scale Sedimentation Dynamics of Colloidal Formulations Through Direct Visualization
}

\section{Shalmali Bapat, Doris Segets}

Submitted date: $26 / 05 / 2020$ - Posted date: 27/05/2020

Licence: CC BY-NC-ND 4.0

Citation information: Bapat, Shalmali; Segets, Doris (2020): Exhibitive Nano-to-Micron Scale Sedimentation Dynamics of Colloidal Formulations Through Direct Visualization. ChemRxiv. Preprint. https://doi.org/10.26434/chemrxiv.12278360.v2

The study of sedimentation behavior of nanoparticle dispersions is important for revealing particle size and colloidal stability characteristics. Quantitative appraisal of real-world colloidal systems in their native state, is key for replacing prevailing empiricism in formulation science by knowledge-based design. Herein, we choose fuel cell inks as one case-example amongst many other possibilities to present a new visualization technique, called Transmittogram. This technique readily depicts the time-resolved settling behavior of solid-liquid dispersions, measured by analytical centrifugation (AC). Although AC enables the causal examination of agglomeration, settling, and creaming behavior of dispersions, along with its consequent effect on structure formation and product properties, the understanding of the main transmission readout is often non-intuitive and complex. Transmittograms are, therefore, the missing link for straightforward data interpretation. First, we illustrate the utility of transmittogram analysis using model silica nanoparticle systems and further validate it against known characteristics of the system. Then, we demonstrate the application of transmittograms to characterize fuel cell inks, showing the strength of the approach in deconvoluting and distilling information to the reader. Finally, we discuss the potential of the technique for routine analysis using analytical centrifugation.

File list (2)

2020-05-24_manuscript_for_preprint.pdf (747.01 KiB) view on ChemRxiv - download file 2020-05-24_supporting_information_for preprint.pdf (1.59 MiB) view on ChemRxiv - download file 


\title{
Exhibitive nano-to-micron scale sedimentation dynamics of colloidal formulations through direct visualization
}

\author{
Shalmali Bapat ${ }^{1}$ and Doris Segets $1,2^{*}$ \\ ${ }^{1}$ Process Technology for Electrochemical Functional Materials, Institute for Combustion and Gas Dynamics - Reactive \\ Fluids (IVG-RF), University of Duisburg-Essen (UDE), Duisburg, Germany \\ ${ }^{2}$ Center for Nanointegration Duisburg-Essen (CENIDE), Duisburg, Germany \\ Keywords: Analytical centrifugation, Sedimentation, Catalyst ink, Stability analysis, Dispersion, Fuel cell
}

\begin{abstract}
The study of sedimentation behavior of nanoparticle dispersions is important for revealing particle size and colloidal stability characteristics. Quantitative appraisal of real-world colloidal systems in their native state, is key for replacing prevailing empiricism in formulation science by knowledge-based design. Herein, we choose fuel cell inks as one caseexample amongst many other possibilities to present a new visualization technique, called Transmittogram. This technique readily depicts the time-resolved settling behavior of solid-liquid dispersions, measured by analytical centrifugation (AC). Although AC enables the causal examination of agglomeration, settling, and creaming behavior of dispersions, along with its consequent effect on structure formation and product properties, the understanding of the main transmission readout is often non-intuitive and complex. Transmittograms are, therefore, the missing link for straightforward data interpretation. First, we illustrate the utility of transmittogram analysis using model silica nanoparticle systems and further validate it against known characteristics of the system. Then, we demonstrate the application of transmittograms to characterize fuel cell inks, showing the strength of the approach in deconvoluting and distilling information to the reader. Finally, we discuss the potential of the technique for routine analysis using AC.
\end{abstract}

Solid in liquid dispersions find widespread applications in energy conversion and storage, foodstuff, pharmaceuticals, and optoelectronics, among others. One such prominent dispersion is catalyst ink, which forms an important building block of polymer electrolyte membrane fuel cells (PEMFCs)..$^{1,2}$ Catalyst ink is a highly complex multi-material dispersion comprising active material, supported on a carbon carrier, and stabilized by a polymeric binder, in a continuous solvent phase. The knowledge of its native dispersion state over time is important for the optimization of ink processing, and consequent large-scale manufacturing of fuel cells. The size and concentration of particles, along with their interactions, affect macroscopic properties such as stability, ${ }^{3,4}$ aggregation, ${ }^{5,6}$ rheology, ${ }^{7-9}$ and electrochemical performance.6,10 However, ink formulation and processing is primarily governed by empiricism and important process-structure-property relationships remain to be adequately explored. $6,9,11$

Recent advances in analytical centrifugation (AC), employing STEP-technology ${ }^{\mathrm{TM}}$ (Space-Time Extinction Profiles) ${ }^{12}$ (shown in Figure 1), permit direct measurement of sedimentation dynamics of particle systems in a facile manner, with low cost and preparation demands. Monitoring the progression of particles in a centrifugal field is attractive for several applications, such as (a) evaluation of processstructure relations, ${ }^{13}$ (b) comparison of emulsifying properties, ${ }^{14,15}$ and (c) assessment of emulsion/dispersion stability. ${ }^{16,17}$ Lately, a handful of studies report the successful use of AC to evaluate ink stability by calculating relative sedimentation times.1,2,18 Collectively, these studies underline the untapped potential of this characterization technique for complex colloids.

Reporting of AC measurement readout is primarily in the form of transmission profiles $(T[r, \tau])$, which are frequently called as fingerprints (see Figure 1). The seminal work by Lerche et al. ${ }^{19}$ serves as a guide to interpret the transmission profiles. In the work described in this letter, we consider two fuel cell ink samples of identical composition as a case demonstration. They were prepared by mixing the carbon-supported platinum powder in a Nafion $®$ dispersion, with 2-propanol and water as solvents. Two different sonication amplitudes of $20 \%$ (sample FCAT_E20) and $50 \%$ (sample FCAT_E50) were used (see supporting information (SI) section 1.2 for details of ink preparation). Figure 2 depicts the transmission fingerprints showing the evolution of relative transmission against the radial position. These plots exemplify the intricacies involved in reading and direct interpretation of fingerprints, for such complex, multimaterial dispersions. For instance, little can be derived regarding the formation of different particle assemblages and their respective sedimentation times. Additionally, the availability of experimental progression time is implicit and qualitative (seen through transitioning curve colors). Besides, several studies that report AC results provide and 


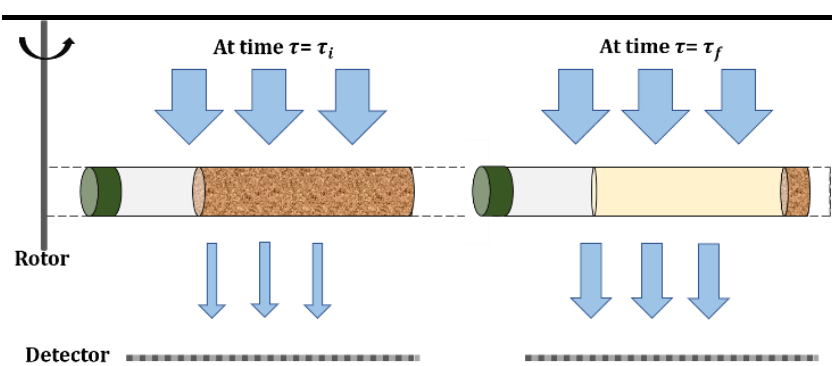

Detector

Transmission fingerprints
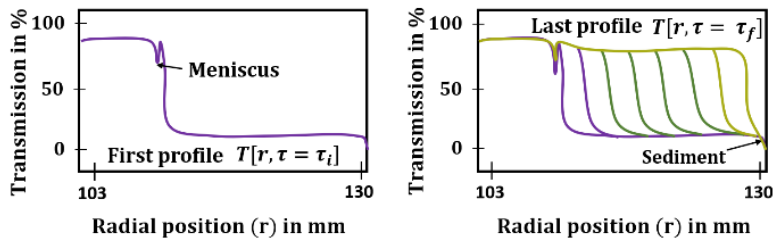

Figure 1. Schematic of an analytical photo centrifuge. Light passes through the sample cell and the instantaneous attenuated transmission signals across the sample length are captured by the CCD detector. First profile (in violet) captured at time $\tau_{i}$ corresponds to unseparated sample. Profiles are captured at fixed time intervals, with the last profile (in yellow) captured at time $\tau_{f}$.

process manually taken images of sample cells (before and after the measurement), juxtaposed to the transmission profiles. This assists the reader to draw meaningful conclusions regarding the sample behavior progressing over time. Despite the utility, it is not always possible to take a snapshot of the sample cell at different time points. Furthermore, the movement of the sample may not be discernible across a couple of centimeters of the sample filling height. Hence, unambiguous identification of particle fractions with distinct sedimentation velocities is impossible without reasonable assumptions or prior system knowledge.

To this end, we introduce a novel visualization technique by transforming the raw data acquired by AC into intuitive visual formats. Such direct visualizations enable easy understanding and interpretation of particle migration dynamics, for expert and inexperienced eyes alike. In a photo
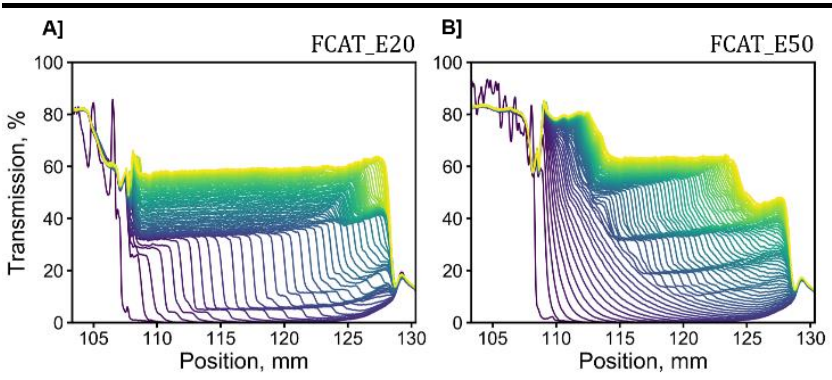

Figure 2. Example of typical transmission fingerprints for dispersions measured using AC. Transmission profiles captured for PEMFC ink samples undergoing centrifugation at 4000 RPM for about 18 hours. Ink samples of identical compositions and concentrations were prepared using ultrasonic bath followed by a probe sonication, with oscillation of [A] $20 \%$ amplitude (sample FCAT_E20) and [B] $50 \%$ amplitude (sample FCAT_E50).

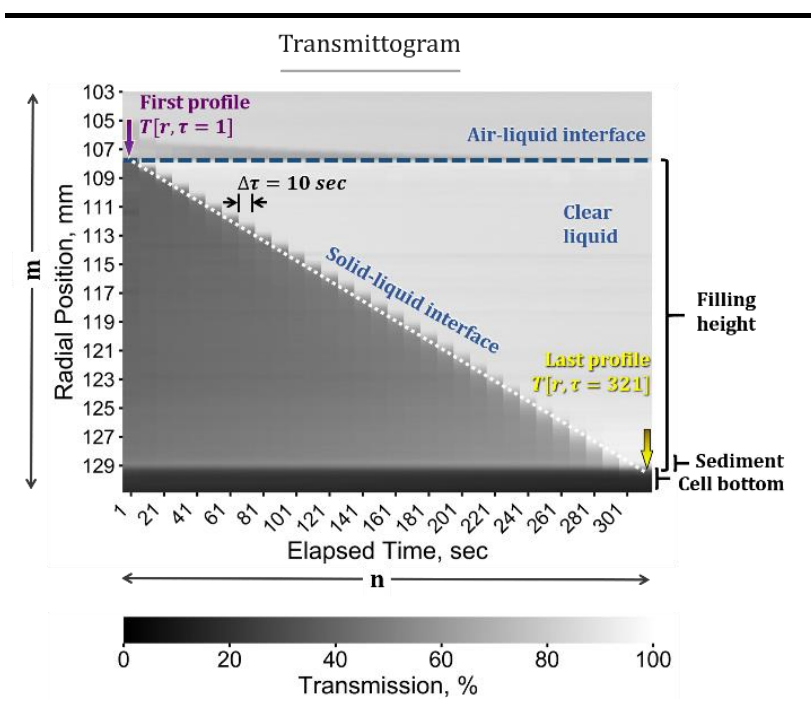

Figure 3. Transmittogram illustrating the time and space resolved change in attenuated transmission values. The radial position of the sample from the rotor center and elapsed time are indicated on the $y$ - and $x$-axis, respectively. A grey value is assigned to instantaneous transmission values $T[r, \tau]$, to construct the heatmap. Underlying data is of monodispersed silica.

centrifuge such as LUMiSizer $®$ (LUM GmbH, Berlin, Germany), the dispersed particles migrate over time in a sample cell, under the influence of gravitation and centrifugal fields. The attenuated optical transmission through the colloidal solution, reaching the array of charged-couple device (CCD) detectors, is captured at fixed time intervals $(\Delta \tau)$. Considering ' $n$ ' number of profiles captured over ' $m$ ' number of spatial points, the resulting data matrix of size ( $m \times n)$ is used to construct a heatmap (see Figure 3). We call such spatio-temporal maps as "Transmittograms". Prior to this work, the term transmittogram has been reported to describe non-thermal infrared vision images. These are generated using a non-destructive technique for testing materials such as paintings, glass, and natural fibers. ${ }^{20,21}$ Additionally, this term has been used by McConville et al. ${ }^{22}$ to describe the transmission-time relationship for nebulization of pharmaceutical dispersions. To the best of our knowledge, the development of such exhibitive maps to depict submicron particle dynamics over space and time is the first of its kind.

To intuitively understand transmittogram plots, parallels can be drawn to image construction. By utilizing all the captured instantaneous transmission values $(T[r, \tau])$ over space (across the sample cell) and time, one image is constructed as schematically illustrated in Figure 3. It is to be noted that the all data used in the scope of this work was acquired using the LUMiSizer $®$ analytical centrifuge. The $\mathrm{x}$ axis of the plot shows the time elapsed from the start of the experiment. The time resolution of the graph depends on the capture interval $(\Delta \tau)$. Smaller capture intervals yield smoother curves for the solid-liquid interface. Note that depending on the chosen time interval, the total elapsed time may vary from experiment to experiment. Currently, the available software paired with AC device (SepView $®$ ) allows the acquisition of profiles at intervals as low as 

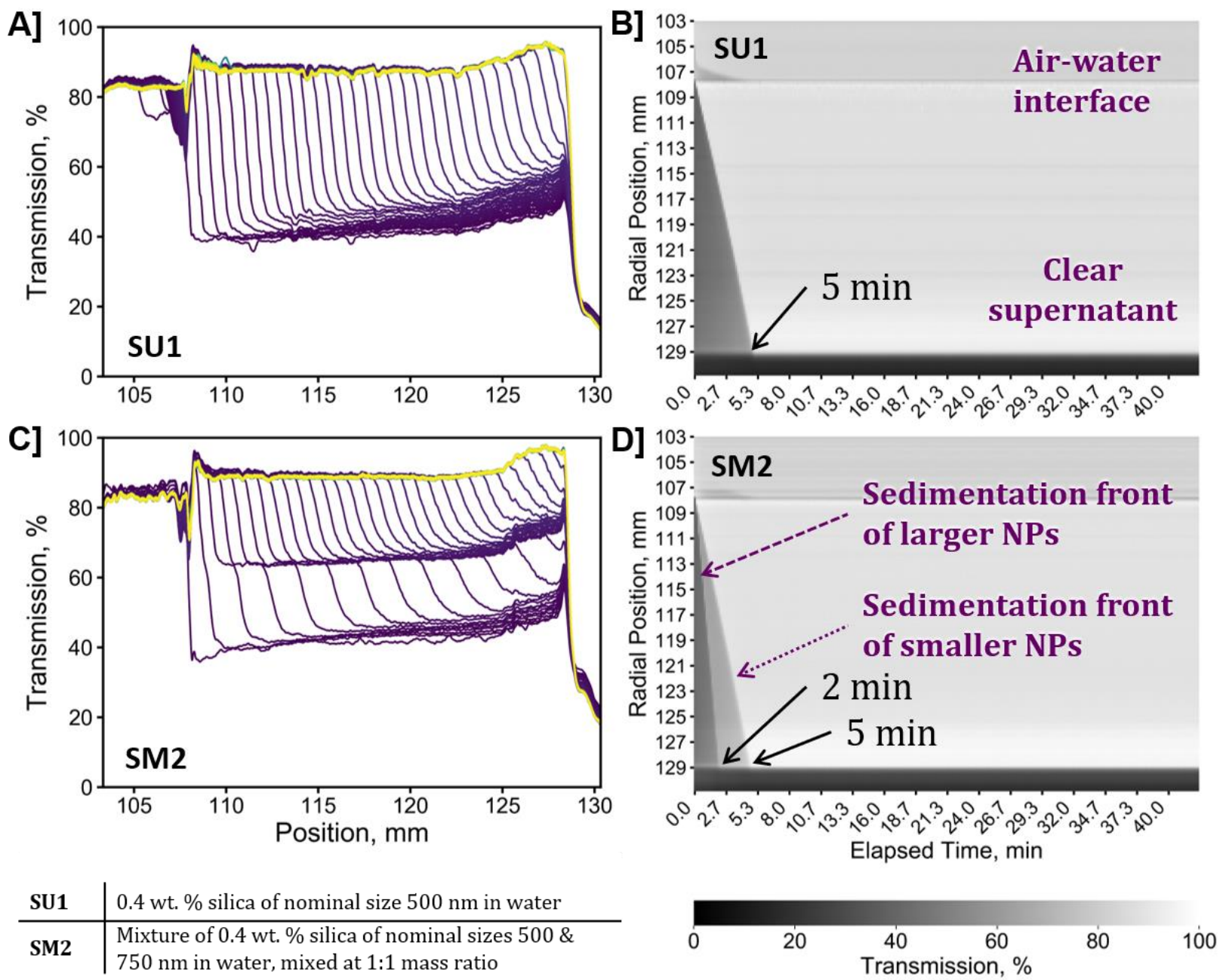

Figure 4. Comparison of fingerprints and transmittograms for silica NPs in water. Data acquired for samples undergoing centrifugation at 2000 RPM for about 40 minutes. [A] Fingerprints of sample SU1, with the [B] corresponding transmittogram. [C] Fingerprints for sample SM2 with the [D] corresponding transmittogram.

5 seconds. The y-axis shows the radial position of the dispersion contents, spanning $103 \mathrm{~mm}$ to $130 \mathrm{~mm}$ from the center of the centrifuge rotor. This is because the filling chamber of the sample cells typically starts at a distance around $103 \mathrm{~mm}$ and ends at $130 \mathrm{~mm}$ from the rotor center. Optical signals are captured at a spatial resolution of $13.37 \mu \mathrm{m}$, across the sample chamber. Here we chose to plot absolute radial distances (instead of the corresponding normalized distances), as these are of assistance in size estimation and radial momentum assessment. Additionally, the radial position values may be counter-intuitive to the increasing $y$-axis values of an exemplary graph. This is because the orientation of a transmittogram plot is chosen in a way to fulfill a facile generalization of human judgment that dense solid matter settles in a "downward" direction. Conversely, visualizing creaming processes with a transmittogram will result in "upward" rising dynamics.

To construct the heatmap, a grey value between 0 to 255 is assigned to the attenuated transmission, acquired across the sample cell, over time (details on the data processing pipeline can be found in SI section 1.6). The darkest color is assigned to the lowest transmission value indicating the highest concentration and the brightest color to the highest transmission value, indicating the lowest concentration. Thus, transmittograms showing changes in transmission profiles are surrogate for change in particle concentrations. This enables the user to instinctively understand complex colloidal migration dynamics, in comparison to more complicated fingerprints.

To emphasize the novelty of the approach, in the following paragraphs, we demonstrate how different types of information can be easily inferred from the visual maps. We illustrate how transmittograms provide a dossier of information regarding the presence of several particle assemblages, with varying sedimentation characteristics. First, we applied the technique to silica NPs as model systems and rationalized our observations against known aspects of the dispersion. Building on these validation studies, we applied the approach to analyze data of PEMFC inks, in their native state without any dilutions. Figure 4A-B (sample SU1) depicts the sedimentation of 0.4 wt. \% silica in water, with a nominal diameter of $500 \mathrm{~nm}$ as given by the manufacturer (see SI section 1.1). Looking at the transmittogram (Figure $4 \mathrm{~B})$, the viewer is instantly furnished with information 

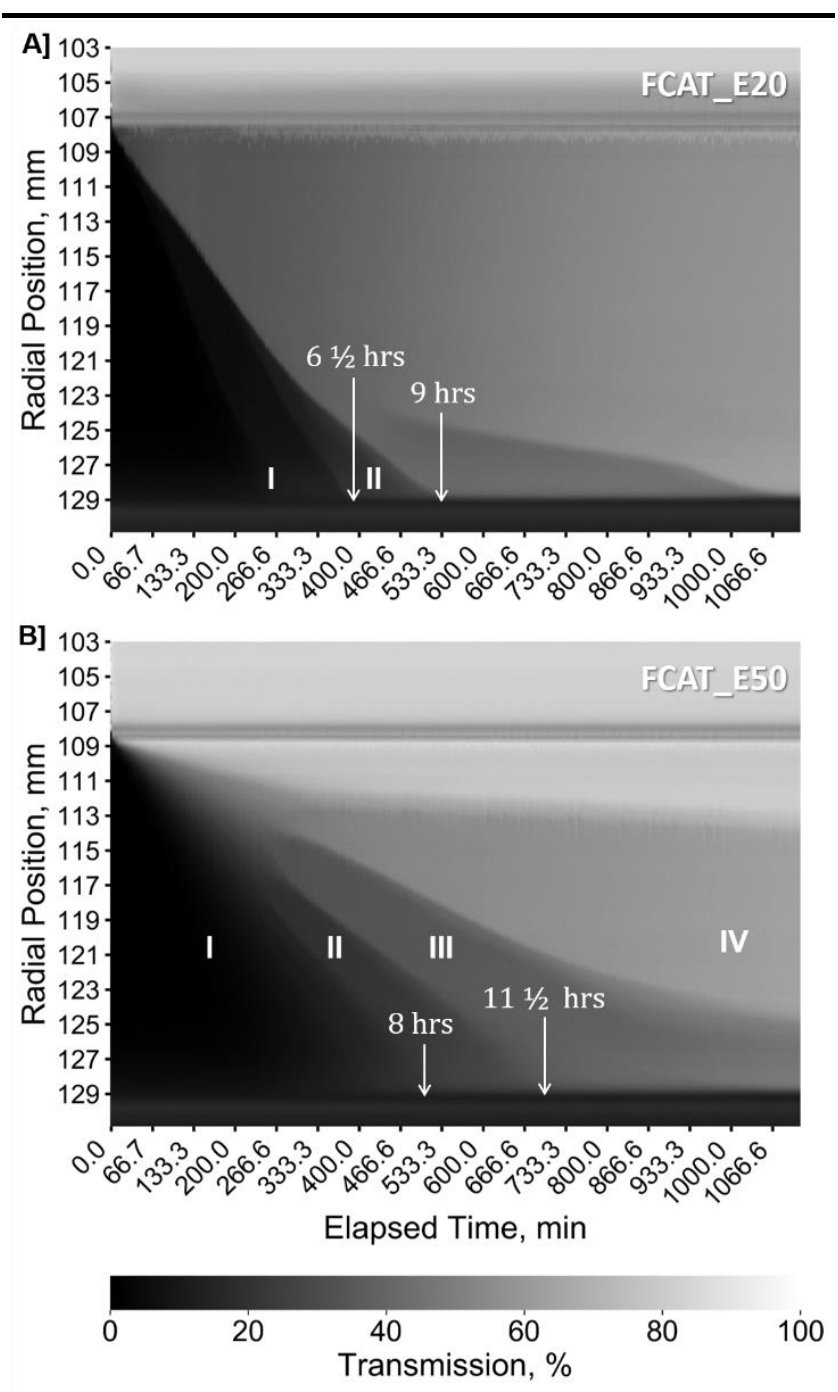

Figure 5. Transmittograms for fuel cell ink samples of identical concentration, prepared using an ultrasonic bath followed by a probe sonication, with oscillation of $[A] 20 \%$ amplitude (sample FCAT_E20) and [B] $50 \%$ amplitude (sample FCAT_E50). Density stratification of agglomerate settling is observed.

regarding the transient state of particle migration at any time $(\tau)$, the progression of the solid-liquid interface until complete settling is achieved, the time taken to sediment completely, i.e., $\sim 5$ minutes and the position of the air-liquid interface (meniscus) around $107 \mathrm{~mm}$. Moreover, the shape of the solid-liquid boundary is indicative of the particle monodispersity as confirmed by manufacturer specifications and SEM imaging (see supporting Figure S1). Figure 4C-D shows the sedimentation of a binary mixture of silica NPs (sample SM2). Here, 0.4 wt. \% silica of nominal diameters 500 and $750 \mathrm{~nm}$ were mixed at a 1:1 mass ratio (see SI section 1.1). Deciphering the fingerprints for SM2 is limited to the eye of an expert. On closer scrutiny of the fingerprints for SM2 (Figure 4C), one can correlate the different manifested shapes of fingerprints sectioning around $65 \%$ transmission, to groups of different sizes.

In contrast, different mobility rates are evident in the transmittogram (Figure 4D) as two different sedimentation fronts can be easily tracked by two color shades. The darker shade (highlighted by the dashed line) indicates the sedimentation front of the larger silica NPs which settle in about 2 minutes while comparatively smaller silica NPs (highlighted by the dotted line) takes about 5 minutes to sediment as shown by the lighter shade. Thus, transmittogram correctly exhibits two differently sized monodispersed components.

Transmittograms not only lend to deducing the behavior of particles in suspension but also provide a size estimate. The sedimentation equivalent diameter $x$ of particles that reach the rotor position $r$ in time $\tau$ can be obtained from the Stoke's law described by ${ }^{23}$

$$
x=\sqrt{\frac{18 \mu \ln \left(\frac{r}{r_{0}}\right)}{\left(\rho_{p}-\rho_{s}\right) \omega^{2} \tau}}
$$

With $\left(\rho_{p}-\rho_{s}\right)$ density difference of particle and solvent in $\mathrm{kg} / \mathrm{m}^{3}$, solvent viscosity $\mu$ in $\mathrm{kg} / \mathrm{m} / \mathrm{s}$, and angular speed $\omega$ in rad/s. As depicted in Figure 4B, the solid-liquid interface for sample SU1 paces from $107 \mathrm{~mm}$ to $129 \mathrm{~mm}$ in about 5 minutes at a rotational speed of 2000 revolutions/minute. The sedimentation equivalent diameter thus calculated from equation 1 for sample SU1 is $390 \mathrm{~nm}$. Particle size analysis in accordance with ISO 13318-2 (SepView@) yields a volume-weighted mean size of $378 \mathrm{~nm}$ (see supporting Figure S2). Likewise, a size estimation for the binary sample SM2 gives sizes of $390 \mathrm{~nm}$ (actual volume-weighted mean size of $378 \mathrm{~nm}$ ) and $587 \mathrm{~nm}$ (actual volume-weighted mean size of $592 \mathrm{~nm}$ ) for particles settling in 5 and 2 minutes, respectively (see Figure 4D).

Having demonstrated how transmittograms are constructed and read for model silica systems, we employed this visualization approach to PEMFC inks. We see that the limitations in the interpretability of fingerprints in Figure 2A-B are overcome by our new approach shown in Figure $5 \mathrm{~A}-\mathrm{B}$. Although specifically studying and comparing different ink processing methods is out of the scope of current work, the aim here is to demonstrate how the direct visualization technique can be leveraged to effectively gather knowledge of the nano-scale dynamics.

Noticeably, transmittograms (Figure 5A-B) provide a global view of the entire sedimentation experiment in contrast to transmission profiles (Figure 2A-B). The heatmaps also fetch additional information about (a) whether bulk sedimentation has been achieved and at what time, (b) density stratification and variations in settling velocities, (c) vicinity of agglomerate groups, and (d) presence of back diffusion. In Figure 5A, FCAT_E20 shows an assemblage of comparatively large size, settling in the course of $\sim 6 \frac{1}{2}$ hours, followed by another assemblage settling by 9 hours. However, there is still a good amount of particulate phase dispersed in the solvent, which tends to settle slowly, suggested by a gradient of gray becoming lighter in shade from left to right (i.e., increase in transmission values from $\sim 30 \%$ to $\sim 60 \%$ ). Furthermore, the bottom right part of Figure 5A shows the occurrence of back-diffusion of the particles over long experiment time (after $\sim 10$ hours).

On the other hand, on using larger oscillation amplitude in sample FCAT_E50, we are able to see discernible changes in the settling pattern. These changes in settling rates, in 
turn, provide insights into the stability of the ink. The four distinct interfaces reveal the existence of at least four different assemblages of varying sedimentation velocity. Assemblage I settles in $\sim 8$ hours, assemblage II in $\sim 11 \frac{1}{2}$ hours. Assemblages III and IV do not completely settle by the end of the experiment running for over 17 hours. Pairwise comparison between FCAT_E20 and FCAT_E50, in Figure 5A-B, permits assessment of sonication settings affecting the dispersion behavior of ink samples. The data suggest that higher oscillation amplitude in FCAT_E50 resulted in partial breaking up of large agglomerates, leading to an increased degree of stabilization. Such direct visual analysis of dispersions using transmittograms hence allows studying the outcome of different processing modes and stabilization strategies on sedimentation mechanisms. Different factors have been reported to have an effect on the ink dispersion behavior, which can be studied on a case-by-case basis. For instance - ultrasonic frequency, ${ }^{5,8}$ intensity of vibrational oscillations, ${ }^{24}$ and temperature. ${ }^{25}$ Illustrative analysis for inks prepared using a different sonication device and measured at higher temperatures are described in SI (see supporting Figure S3).

Thus far, the results provide important insights into the number of assemblage fractions and their characteristic sedimentation times. These insights can be exploited, first to compare differently processed inks, and second, to understand how ink properties affect the final PEMFC performance. Although both fingerprints and transmittograms rely on the same raw data source, the latter noticeably enhances the utility of the AC technique by revealing properties of complex ink formulations, which could not be accessed with the former.

Developing a time-lapsed graph for complete particle concentration gradient has potential benefits for several other real-world particulate systems exhibiting polydispersity and multimodality. ${ }^{26}$ As proof of concept, showing the usefulness of transmittograms to study other complex colloidal fluids, similar analysis was performed for whole human blood (see supporting Figure S4) and orange juice (see supporting Figure S5). Moreover, the concept of generating such visual maps can be extended to data acquired from other sedimentation-based particle characterization methods such as Turbiscan ${ }^{27}$ Having discussed the construction of transmittograms and its potential use in deducing qualitative and quantitative aspects about multi-material formulations, the final section of the paper addresses some practical considerations.

It is important to bear in mind recommendations of standard operating protocols, for instance, to avoid filling the sample to the brim. ${ }^{28}$ Since the CCD detector is positioned between the radial distance of $103 \mathrm{~mm}$ to $130 \mathrm{~mm}$, edge effects may be dominant in photon detection and transfer. Hence in reading a transmittogram, the top and bottom extreme edges should be interpreted with care. Besides, at times the instrument provides raw data with implausible relative transmission values $(T[r, \tau]<0$ and $T[r, \tau]>100)$ due to electronic aberrations, care must be taken in parsing such values as it may lead to biased interpretation of the transmittogram. Herein, such implausible values were replaced appropriately (for example, negative values were replaced by 0.1). More sophisticated statistical imputing methods can also be applied.

Secondly, for the transmittograms discussed so far, a monotonically increasing color map was assigned linearly to transmission values between 0 and 100 . However, the density of transmission values $(T[r, \tau])$ can be very high in a certain range, causing a large set of values mapped to the same (nearby) colors. For the discussed approach to be usable, the generated image should be visually informative for us to perceive it properly. Plots with a high level of contrast to distinguish solute (foreground) from solvent (background) will tend to be more informative. To alleviate the issues with screen contrast, to achieve the best visual perception, we employ different intelligent color assignment techniques. Firstly, color values can be mapped in a non-linear fashion. Likewise, data normalization and transformations (e.g., power transformation) can spread out the distribution of transmission values, for enhanced color mapping (see supporting Figure S6B-C for non-linear color mapping). Taking this a step further, specific colors can also be mapped to different user-defined transmission intervals. To intelligently choose optimum intervals, we implemented a quantile-based approach, to divide the array of transmission values and optimally assign different color breaks (see supporting Figure S6D).

Lastly, as with any image, color perception of the eye, screen contrast, or print quality can make image viewing a radically different experience from person to person. Here in the case of transmittograms the sample migration can be imperceptible to the human eye in some cases. When transmission values are distributed as a unimodal Gaussian, over a small range (see supporting Figure S7A-D), they are mapped to similar shades of gray. This makes effective thresholding to distinguish a solid-liquid interface challenging. This problem can be alleviated with carefully curated, perceptually distinct color maps tailored to offer improved contrast. Additionally, the choice of colormaps can be made based on different color principles and sample appearance. Diverging colormaps can also be employed to make a clear distinction between clear liquid and space above the sample filling height. In this case, the diverging point color is assigned to transmission values around the solvent meniscus. Such methods for smart color mapping not only improve the readability of the plots but also reveal confounded colloidal strata (see supporting Figure S8).

In summary, our work introduces a new direct visualization technique by plotting heatmaps of attenuated optical signals captured using AC. By utilizing complete available raw data, we generate transmittograms to simplify interpretations of the labyrinthine transmission profiles. We believe this could be an important part of routine analysis for any sample characterized using an analytical centrifuge such as LUMiSizer $®$. Using transmittograms we can derive qualitative and quantitative characteristics that are together important to establish the physical reality of complex colloidal systems. To demonstrate the utility of the method, we first applied it to simple and well-known particle systems. Building on what is known through the interpretation of simple systems, we increased the complexity by investigating fuel cell inks. We showed how one can distill relevant information from the plots and how transmittograms can be used to study dispersion behavior. Furthermore, on 
deconvoluting and deducing the state of dispersions, one can rationalize the visual cues in a statistical framework. Moreover, the characteristics and observations obtained from transmittograms can aid in establishing informed assumptions and boundary conditions for bottom-up mathematical modeling strategies. In pursuit of revealing the nano-and micron-scale dynamics of such particulate systems, we believe our transmittogram approach is a significant addition to the existing characterization toolbox.

\section{ASSOCIATED CONTENT}

Supporting information describing materials, methods, and results. Materials and methods include sample preparation methods, electron microscopy imaging, analytical centrifugation characterizations. Results include SEM micrographs, size analysis, transmittogram analysis for a set of PEMFC inks, whole human blood, and orange juice, along with plots illustrating color mapping considerations.

\section{AUTHOR INFORMATION}

\section{Corresponding Author}

Doris Segets - Process Technology for Electrochemical Functional Materials, Institute for Combustion and Gas DynamicsReactive Fluids (IVG-RF), University of Duisburg-Essen, Duisburg 47057, Germany; orcid.org/0000-0003-3102-2934; Email: doris.segets@uni-due.de

\section{Author Contributions}

S.B. developed the idea, implemented the method, prepared samples, performed experiments, analyzed data, developed code to create custom data processing workflows and wrote the manuscript. D.S. designed and supervised the project, contributed to interpretation of findings and manuscript writing.

\section{Notes}

The authors declare no competing financial interests.

\section{ACKNOWLEDGMENT}

S.B gratefully acknowledges scholarship from International Max-Planck Research School (IMPRS) for Interface Controlled Materials for Energy Conversion, under the SurMat program. The authors acknowledge NPPT lab (IVG) for imaging facility and Dr. Fatih Özcan (IVG-RF) for SEM image acquisition of silica particles. The authors would like to thank Dr. Hans Orthner (IVG-RF) for providing sonication device and Ms. Sabrina Fricke from the hydrogen and fuel cell center (ZBT GmbH) for assistance in ink preparation.

\section{REFERENCES}

(1) Thoma, M.; Lin, W.; Hoffmann, E.; Sattes, M. M.; Segets, D.; Damm, C.; Peukert, W. Simple and Reliable Method for Studying the Adsorption Behavior of Aquivion Ionomers on Carbon Black Surfaces. Langmuir 2018, 34 (41), 12324-12334. DOI: 10.1021/acs.langmuir.8b02726.

(2) Hoffmann, E.; Zhang, S.; Thoma, M.; Damm, C.; Peukert, W. Formulation of Carbon Black-Ionomer Dispersions for Thin Film Formation in Fuel Cells. Particuology 2019, 44, 7-21. DOI: 10.1016/j.partic.2018.08.001.

(3) Shukla, S.; Bhattacharjee, S.; Weber, A. Z.; Secanell, M. Experimental and Theoretical Analysis of Ink Dispersion Stability for Polymer Electrolyte Fuel Cell Applications. J. Electrochem. Soc. 2017, 164 (6), F600-F609. DOI: 10.1149/2.0961706jes.
(4) Dixit, M. B.; Harkey, B. A.; Shen, F.; Hatzell, K. B. Catalyst Layer Ink Interactions That Affect Coatability. J. Electrochem. Soc. 2018, 165 (5), F264-F271. DOI:10.1149/2.0191805jes.

(5) Pollet, B. G. The Use of Ultrasound for the Fabrication of Fuel Cell Materials. Int. J. Hydrogen Energy 2010, 35 (21), 1198612004. DOI:10.1016/j.ijhydene.2010.08.021.

(6) Wang, M.; Park, J. H.; Kabir, S.; Neyerlin, K. C.; Kariuki, N. N.; Lv, H.; Stamenkovic, V. R.; Myers, D. J.; Ulsh, M.; Mauger, S. A. Impact of Catalyst Ink Dispersing Methodology on Fuel Cell Performance Using In-Situ X-Ray Scattering. ACS Appl. Energy Mater. 2019, 2 (9), 6417-6427. D0I:10.1021/acsaem.9b01037.

(7) Gallo Stampino, P.; Cristiani, C.; Dotelli, G.; Omati, L.; Zampori, L.; Pelosato, R.; Guilizzoni, M. Effect of Different Substrates, Inks Composition and Rheology on Coating Deposition of Microporous Layer (MPL) for PEM-FCs. Catal. Today 2009, 147 (SUPPL.), 30-35. DOI:10.1016/j.cattod.2009.07.023.

(8) Pollet, B. G.; Goh, J. T. E. The Importance of Ultrasonic Parameters in the Preparation of Fuel Cell Catalyst Inks. Electrochim. Acta 2014, 128, 292-303. DOI:10.1016/j.electacta.2013.09.160.

(9) Khandavalli, S.; Park, J. H.; Kariuki, N. N.; Myers, D. J.; Stickel, J. J.; Hurst, K.; Neyerlin, K. C.; Ulsh, M.; Mauger, S. A. Rheological Investigation on the Microstructure of Fuel Cell Catalyst Inks. ACS Appl. Mater. Interfaces 2018, 10 (50), 4361043622. DOI:10.1021/acsami.8b15039.

(10) Bredol, M.; Szydło, A.; Radev, I.; Philippi, W.; Bartholomäus, R.; Peinecke, V.; Heinzel, A. How the Colloid Chemistry of Precursor Electrocatalyst Dispersions Is Related to the Polymer Electrolyte Membrane Fuel Cell Performance. J. Power Sources 2018, 402 (June), 15-23. DOI:10.1016/j.jpowsour.2018.09.005.

(11) Mauger, S. A.; Neyerlin, K. C.; Yang-Neyerlin, A. C.; More, K. L.; Ulsh, M. Gravure Coating for Roll-to-Roll Manufacturing of Proton-Exchange-Membrane Fuel Cell Catalyst Layers. J. Electrochem. Soc. 2018, 165 (11), F1012-F1018. DOI:10.1149/2.0091813jes.

(12) Detloff, T.; Sobisch, T.; Lerche, D. Particle Size Distribution by Space or Time-Dependent Extinction Profiles Obtained by Analytical Centrifugation (Concentrated Systems). Powder Technol. 2007, 174 (1-2), 50-55. DOI:10.1016/j.powtec.2006.10.021.

(13) Mert, B. Using High-Pressure Microfluidization to Improve Physical Properties and Lycopene Content of Ketchup Type Products. J. Food Eng. 2012, 109 (3), 579-587.

(14) Wanyi, W.; Lu, L.; Zehan, H.; Xinan, X. Comparison of Emulsifying Characteristics of Different Macromolecule Emulsifiers and Their Effects on the Physical Properties of Lycopene Nanoemulsions. J. Dispers. Sci. Technol. 2020, 41 (4), 618-627. DOI:10.1080/01932691.2019.1610421.

(15) Dammak, I.; Sobral, P. J. do A. Effect of Different Biopolymers on the Stability of Hesperidin-Encapsulating 0/W Emulsions. J. Food Eng. 2018, 237, 33-43. DOI:10.1016/j.jfoodeng.2018.05.004.

(16) Hoffmann, W. Potential, and Restrictions of Photocentrifugation for Determining the Emulsion Stability of Melted Spreadable Processed Cheese. Dairy Sci. Technol. 2016, 96 (2), 251-259. DOI:10.1007/s13594-015-0252-3.

(17) Li, X.; Wang, X.; Xu, D.; Cao, Y.; Wang, S.; Wang, B.; Wang, C.; Sun, B. Influence of Calcium-Induced Droplet Heteroaggregation on the Physicochemical Properties of Oppositely Charged Lactoferrin Coated Lutein Droplets and Whey Protein IsolateCoated DHA Droplets. Food Funct. 2017, 8 (8), 2748-2759. DOI:10.1039/c7fo00657h.

(18) Süß, S.; Sobisch, T.; Peukert, W.; Lerche, D.; Segets, D. Determination of Hansen Parameters for Particles: A Standardized Routine Based on Analytical Centrifugation. Adv. Powder Technol. 2018, 29 (7), 1550-1561. DOI: 10.1016/j.apt.2018.03.018.

(19) Lerche, D.; Sobisch, T. Evaluation of Particle Interactions by in Situ Visualization of Separation Behaviour. Colloids Surfaces 
A Physicochem. Eng. Asp. 2014, 440, 122-130. DOI:10.1016/j.colsurfa.2012.10.015.

(20) Kushel, D. A. Applications of Transmitted Infrared Radiation to the Examination of Artifacts. Stud. Conserv. 1985, 30 (1), 1-10. DOI:10.1179/sic.1985.30.1.1.

(21) Liu, Z.; Ukida, H.; Ramuhalli, P.; Editors, K. N. Advances in Computer Vision and Pattern Recognition Integrated Imaging and Vision Techniques for Industrial Inspection Advances and Applications. 2010, 21(5), 597-599.

(22) McConville, J. T.; Carvalho, T. C.; Narain, N. R.; McCook, J. P. Inhalable Pharmaceutical Compositions, 2014.

(23) Lerche, D.; Sobisch, T.; Detloff, T.; Babick, F.; Stintz, M. Method and Device for Characterisation of Multiple Samples of One or Various Dispersions, U.S. Patent 8,265,882, September 11, 2012.

(24) Retamal Marín, R. R.; Babick, F.; Lindner, G. G.; Wiemann, M.; Stintz, M. Effects of Sample Preparation on Particle Size Distributions of Different Types of Silica in Suspensions. Nanomaterials 2018, 8 (7). DOI:10.3390/nano8070454.

(25) Tzanakis, I.; Lebon, G. S. B.; Eskin, D. G.; Pericleous, K. Investigation of the Factors Influencing Cavitation Intensity during the Ultrasonic Treatment of Molten Aluminium. Mater. Des. 2016 90, 979-983. DOI:10.1016/j.matdes.2015.11.010.

(26) Anderson, W.; Kozak, D.; Coleman, V. A.; Jämting, Å. K.; Trau, M. A Comparative Study of Submicron Particle Sizing Platforms: Accuracy, Precision, and Resolution Analysis of Polydisperse Particle Size Distributions. J. Colloid Interface Sci. 2013, 405, 322-330. DOI:10.1016/j.jcis.2013.02.030.

(27) Mengual, O.; Meunier, G.; Cayre, I.; Puech, K.; Snabre, P. Characterisation of Instability of Concentrated Dispersions by a New Optical Analyser: The TURBISCAN MA 1000. Colloids Surfaces A Physicochem. Eng. Asp. 1999, 152 (1-2), 111-123. DOI:10.1016/S0927-7757(98)00680-3.

(28) LUM GmbH. Manual LUMiSizer / LUMiFuge Version 3.8.1. 2018. 


\section{Exhibitive nano-to-micron scale sedimentation dynamics of colloidal formulations through direct visualization}

Shalmali Bapat ${ }^{1}$ and Doris Segets ${ }^{1,2 *}$

${ }^{1}$ Process Technology for Electrochemical Functional Materials, Institute for Combustion and Gas Dynamics Reactive Fluids (IVG-RF), University of Duisburg-Essen (UDE), Duisburg, Germany

${ }^{2}$ Center for Nanointegration Duisburg-Essen (CENIDE), Germany

Address correspondence to doris.segets@uni-due.de

\section{Supporting Information}

\section{Materials and methods}

\subsection{Model system of silica nanoparticles (NPs)}

Spherical silica particles of size, $150 \mathrm{~nm}, 200 \mathrm{~nm}, 500 \mathrm{~nm}, 750 \mathrm{~nm}$ were purchased from Sigma Aldrich. These particles were suspended in ultrapure water $(18.2 \mathrm{M} \Omega . \mathrm{cm})$ to make separate dispersions. Three dispersion systems were prepared namely, SU1, SM2 and SM4 (see Table S1). The NP solutions were introduced into an ultrasonic bath (Elmasonic S10, Elma Schmidbauer $\mathrm{GmbH}$ ) for 30 minutes, for adequate mixing.

Table S1: Silica nanoparticle (NP) dispersion composition.

\begin{tabular}{cccc}
\hline $\begin{array}{c}\text { Sample } \\
\text { name }\end{array}$ & Dispersed solid & $\begin{array}{c}\text { Size (nm) as per } \\
\text { manufacturer }\end{array}$ & Dispersion composition \\
\hline SU1 & Unimodal silica & 500 & 0.4 wt. $\%$ in water \\
SM2 & Bimodal silica & 500 and 750 & $0.4 \mathrm{wt}$ \% in water at $1: 1$ mass ratio \\
SM4 & 4-modal silica & $150,200,500,750$ & $5 \mathrm{wt} \%$ in water at $4: 3: 2: 1$ mass ratio \\
\hline
\end{tabular}

\subsection{Polymer electrolyte membrane fuel cell (PEMFC) catalyst inks}

The catalyst ink was prepared by mixing platinum/carbon powder (HISPEC@ 4000, 40 wt. \% Pt/C, Alfa Aesar), Nafion ${ }^{\circledR}$ dispersion (D521,1100 EW at 5 wt. \%, Ion Power), ultrapure water (18.2 $\mathrm{M} \Omega . \mathrm{cm})$ and 2-propanol ( $\geq 99.0 \%$ purity, VWR international) at desired ionomer/catalyst and solvent volume ratio. Inks of identical composition were prepared by four different dispersion procedures. The mixtures were grouped based on dispersion preparation procedures. In the first group, ink samples FCAT_E20 and FCAT_E50 were mixed 
using a sonication bath (Elmasonic S10) for 30 minutes followed by 15 minutes of a probe sonication (UP300ST, Hielscher Ultrasonics $\mathrm{GmbH}$ ) at amplitudes of $20 \%$ and $50 \%$ (2 minutes $\mathrm{ON}$ and 1 minute OFF) respectively. An ice bath was used to avoid the overheating of the probe. In the second group, ink samples FCAT_U10 and FCAT_U70 were prepared by mixing in a sonication bath (USC 500 TH, VWR International) for 30 minutes, followed by 15 minutes of a probe sonication (Sonifier 450, Branson Ultrasonics) at amplitudes of $10 \%$ and $70 \%$ ( 2 minutes $\mathrm{ON}$ and 1 minute OFF) respectively. An ice bath was used to avoid the overheating of the probe.

\subsection{Complex fluid systems}

Whole Human blood was purchased from VWR international and orange juice from a local supermarket. They were measured as received using an analytical centrifuge.

\subsection{SEM imaging}

SEM was performed using a JEOL JSM-7500F microscope. Acceleration voltage was set to $5 \mathrm{kV}$ and the secondary electron detector was used. Pure silica powders were directly put on a SEM adhesive tape.

\subsection{Analytical centrifugation (AC)}

AC measurements were performed with a LUMiSizer® 651 (LUM GmbH, Berlin, Germany). For a typical AC run, a near-infrared wavelength of $870 \mathrm{~nm}$ was used. Polycarbonate cells (LUM GmbH, Berlin, Germany) with an optical path length of $2 \mathrm{~mm}$ were used for all measurements. The sample cells were filled with $420 \mu \mathrm{l}$ of sample. Sample cells filled with the silica suspensions and fuel cell inks were inserted into the rotor immediately after the ultrasonication step. Whole human blood and orange juice were refrigerated overnight on receiving, followed by direct measurement. Table S2 summarizes the analytical centrifuge test settings. 
Table S2: AC test matrix.

\begin{tabular}{cccc}
\hline Sample code & Temperature & Capturing time & Rotational speed (RPM) \\
\hline SU1 & $25^{\circ} \mathrm{C}$ & $10 \mathrm{~s}$ & 2000 \\
SM2 & $25^{\circ} \mathrm{C}$ & $10 \mathrm{~s}$ & 2000 \\
SM4 & $25^{\circ} \mathrm{C}$ & $10 \mathrm{~s}$ & Varying from 500 to 3000 \\
\hline FCAT_E20 & $7{ }^{\circ} \mathrm{C}$ & $200 \mathrm{~s}$ & 4000 \\
FCAT_E50 & $7{ }^{\circ} \mathrm{C}$ & $200 \mathrm{~s}$ & 4000 \\
FCAT_U10 & $25^{\circ} \mathrm{C}$ & Varying from 5 to $600 \mathrm{~s}$ & 4000 \\
FCAT_U70 & $25^{\circ} \mathrm{C}$ & Varying from 5 to $600 \mathrm{~s}$ & 4000 \\
\hline Human blood & $7^{\circ} \mathrm{C}$ & $5 \mathrm{~s}$ & Varying from 200 to 3200 \\
\hline Orange juice & $7{ }^{\circ} \mathrm{C}$ & $10 \mathrm{~s}$ & Varying from 200 to 3200 \\
\hline
\end{tabular}

\subsection{Data processing methods for constructing transmittograms}

Scientific libraries in Python language were used to construct the transmittograms. Numerical processing was performed with Numpy ${ }^{1}$ and Pandas ${ }^{2}$ and plotting was done using Matplotlib. ${ }^{3}$ 


\section{Results}

\subsection{SEM imaging of silica nanoparticles}

A]

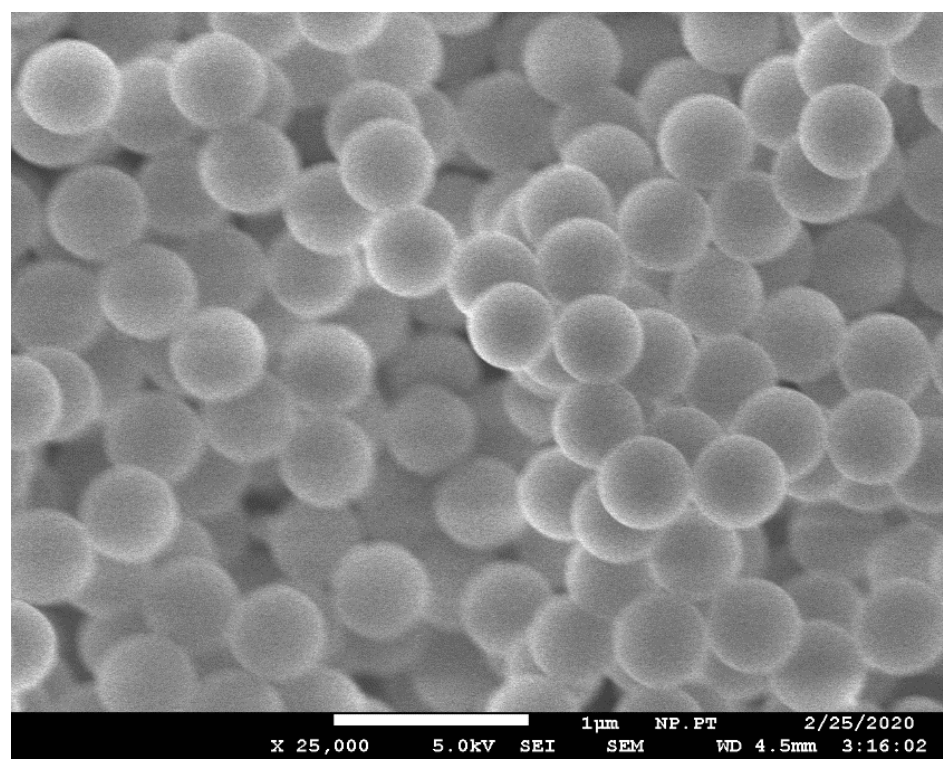

B]

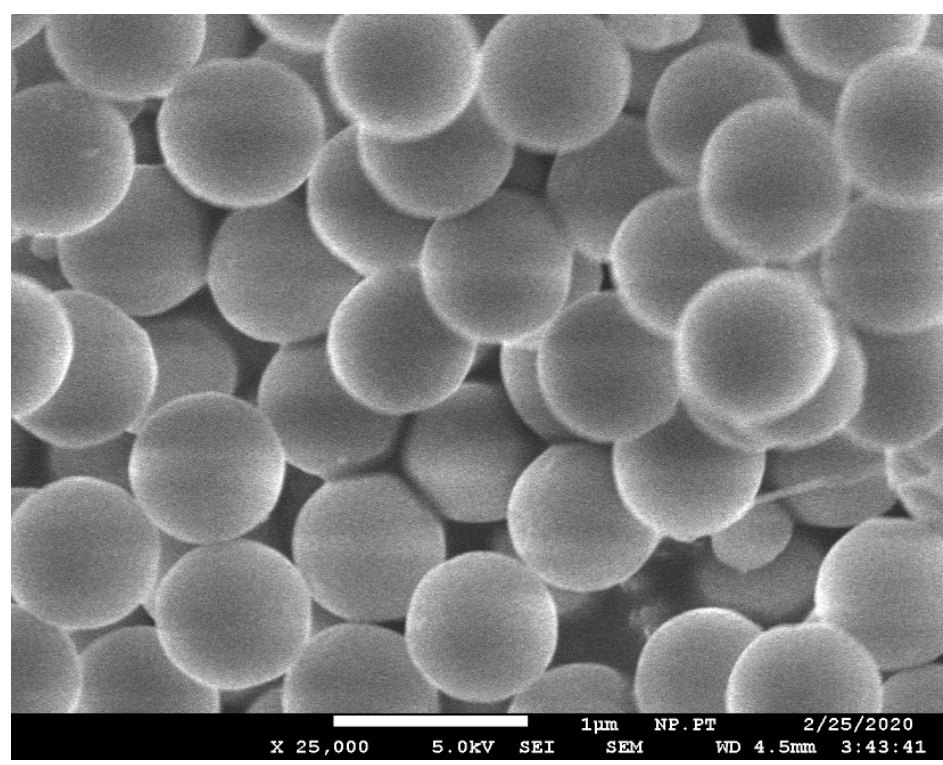

Figure S1: Scanning electron microscopy (SEM) micrographs showing the spherical shape of (A) pure silica of nominal size $500 \mathrm{~nm}$ and (B) pure silica of nominal size $750 \mathrm{~nm}$ at a magnification of 25000 . 


\subsection{Size estimation of silica NPs using AC software (SepView $®)$}

Figure S2 shows the volume-based particle size distribution for silica NPs. The 'constant position' mode in the analytical centrifuge device software SepView® was used for the analysis. The chosen radial positions were $123 \mathrm{~mm}, 125 \mathrm{~mm}$, and $127 \mathrm{~mm}$. It is to be noted that manufacturer specified sizes were $500 \mathrm{~nm}$ and $750 \mathrm{~nm}$.

A]

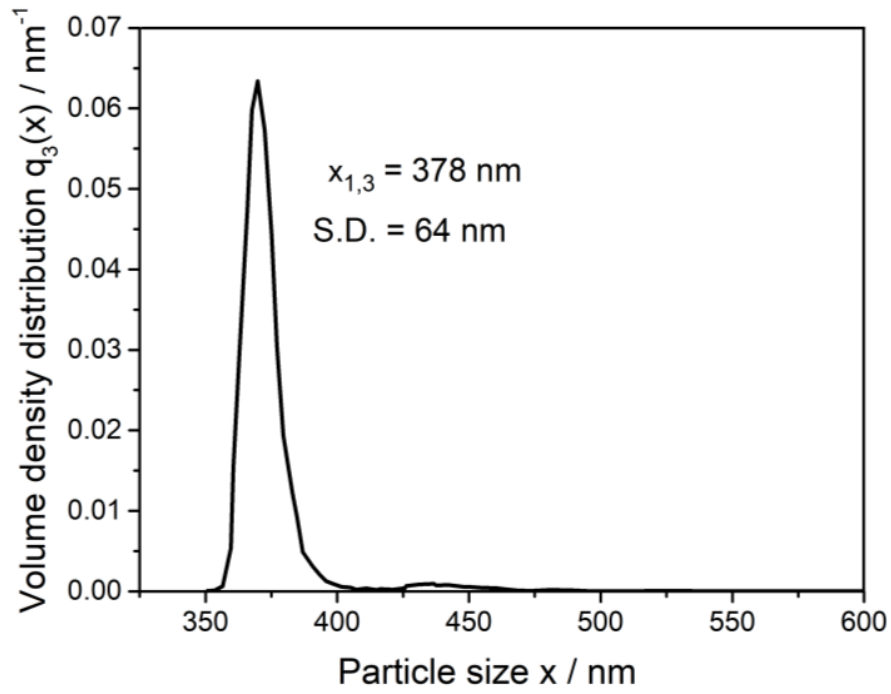

B]

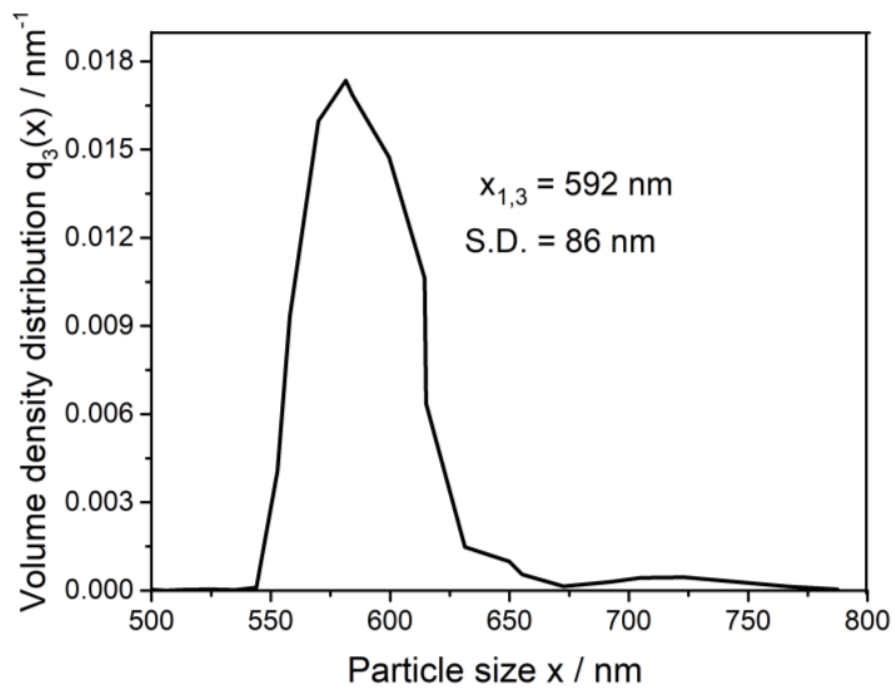

Figure S2: Particle size analysis of silica NPs obtained from analytical centrifugation. (A) Volume weighted mean size of $378+/-64 \mathrm{~nm}$ obtained for manufacture specified $500 \mathrm{~nm}$ silica NPs. (B) Volume weighted mean size of $592+/-86 \mathrm{~nm}$ obtained for manufacture specified $750 \mathrm{~nm}$ silica NPs. 


\subsection{Transmittograms for fuel cell inks prepared by different probe sonication instrument}

Figures S3A and S3B show the transmittograms for catalyst inks prepared using a probe sonication instrument at $70 \%$ and $10 \%$ oscillation amplitude, respectively (FCAT_U70 and FCAT_U10). The sonication device used herein is of a different build compared to the samples FCAT_E20 and FCAT_E50 shown in the main text. For the analytical centrifugation measurement, the capture time interval $(\Delta \tau)$ ramp up from 5 to $25,100,200$, up to 600 seconds. One can instantly pinpoint the effect of ramping capture intervals as the sedimentation fronts show "shoulder breaks". These breaks explain the effect of instrument acquisition settings and operating conditions. Figure S3B shows stratification into at least three assemblages. Assemblage I settle twice as fast as assemblage II and about 5 times as fast as assemblage III. Figure S3C shows the calculation for sedimentation rates of different assemblages. 
A]

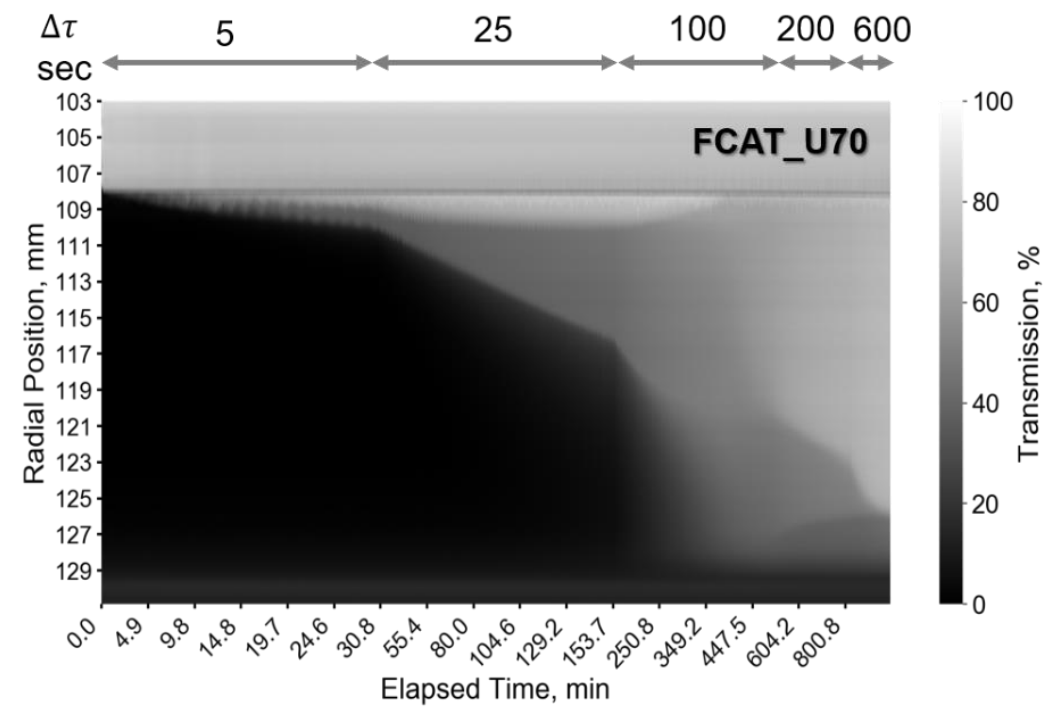

B]

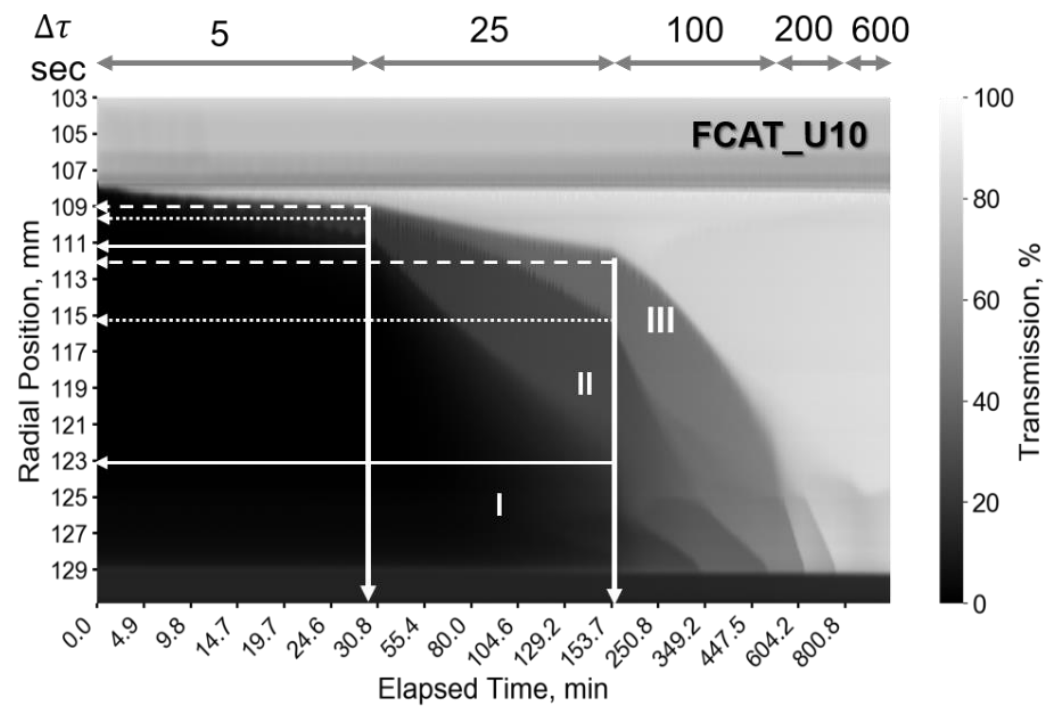

C]

\begin{tabular}{|c|c|c|c|c|c|}
\hline Assemblage & $\begin{array}{l}\text { Initial position } \\
\text { (r0) (mm) }\end{array}$ & $\begin{array}{c}\text { Final position }(\mathrm{r}) \\
(\mathrm{mm})\end{array}$ & $\begin{array}{l}\text { Total displacement } \\
\text { (r-r0) (mm) }\end{array}$ & Total time (min) & $\begin{array}{l}\text { Average speed } \\
\text { (sedimentation } \\
\text { rate) }(\mathrm{mm} / \mathrm{min})\end{array}$ \\
\hline I & 111 & 123 & 12 & 125 & 0.096 \\
\hline II & 109.5 & 115.5 & 6 & 125 & 0.048 \\
\hline III & 109 & 111.5 & 2.5 & 125 & 0.02 \\
\hline
\end{tabular}

Figure S3: Transmittograms for catalyst inks. Sample measurements performed using analytical centrifuge with a rotor temperature of $25{ }^{\circ} \mathrm{C}$, at $4000 \mathrm{RPM}$, and variable capture intervals. (A) Transmittogram of sample FCAT_U70 prepared at $70 \%$ amplitude. (B) Transmittogram of sample FCAT_U10 prepared at $10 \%$ amplitude. (C) Different sedimentation rates of three assemblages were calculated using by tracking sedimentation front in the transmittogram for FCAT_U10. 


\subsection{Transmittogram for whole human blood}

Figure S5A-B shows the fingerprints and the corresponding transmittogram of whole human blood. The centrifugation speed was ramped from 200 RPM to 3200 RPM. At least three groups of particles moving at different velocities can easily be seen from the transmittogram.

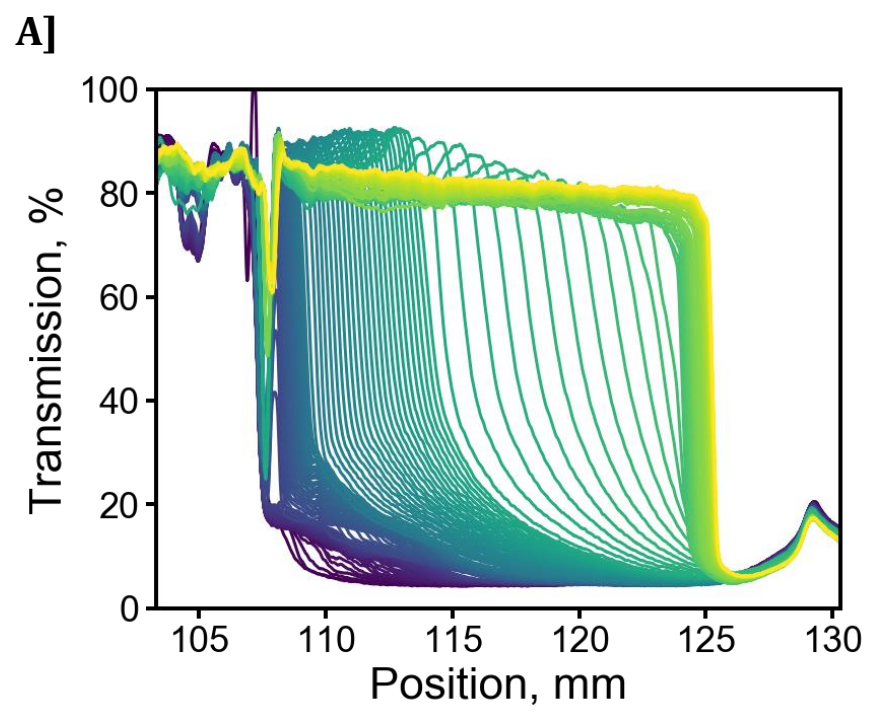

B]

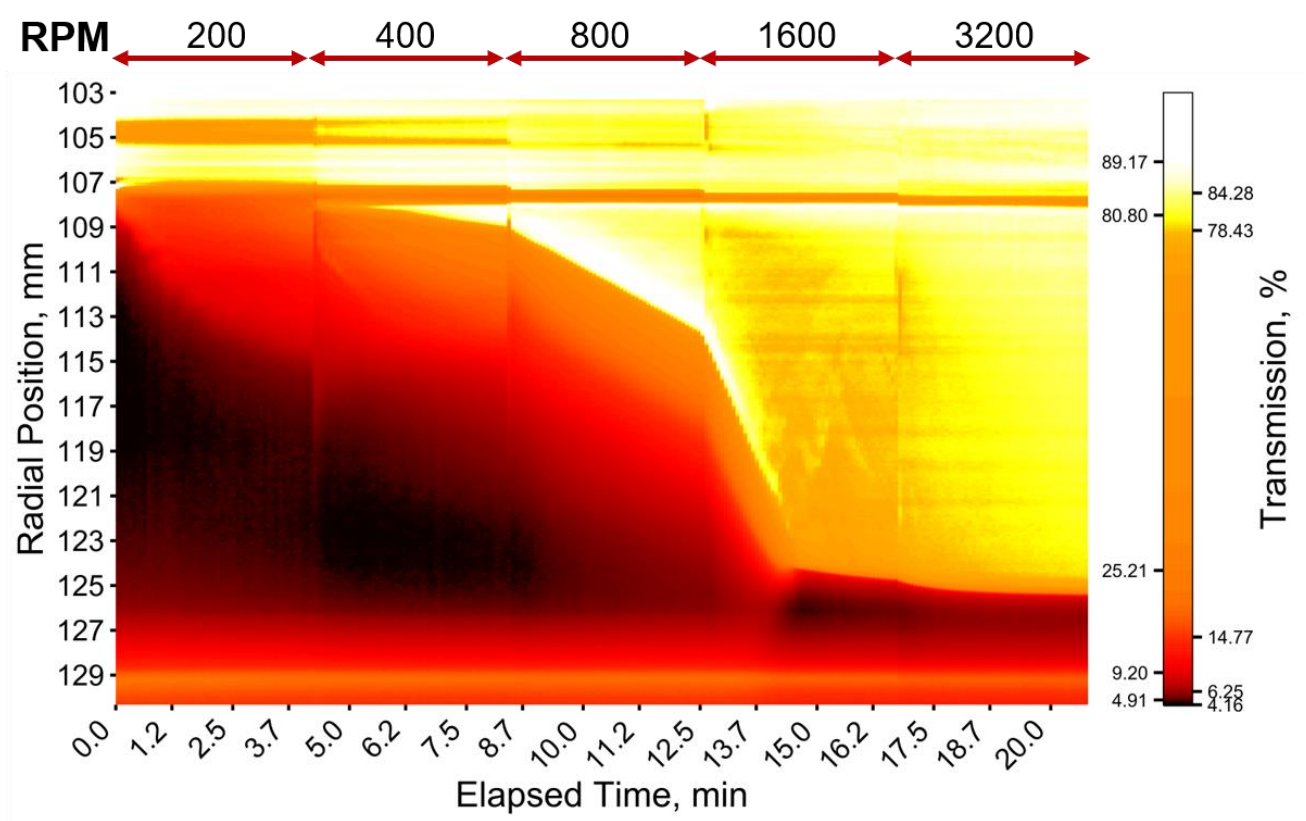

Figure S4: (A) Fingerprints for whole human blood measured using analytical centrifuge, at constant time interval of 5 seconds, varying speed from $200 \mathrm{RPM}$ to $3200 \mathrm{RPM}$ at $7{ }^{\circ} \mathrm{C}$. (B) Transmittogram for whole human blood, employing automatic quantile color breaks approach for improved visual contrast and better visualization of sediment stratification. 


\subsection{Transmittogram for orange juice}

Figure S5A-B shows the fingerprints and the corresponding transmittogram for an orange juice sample. The centrifugation speed was ramped from 200 RPM to 3200 RPM. With the help of a transmittogram, one can easily pinpoint the time at which the speed was changed causing change in rate of sedimentation, which is otherwise not directly accessible.

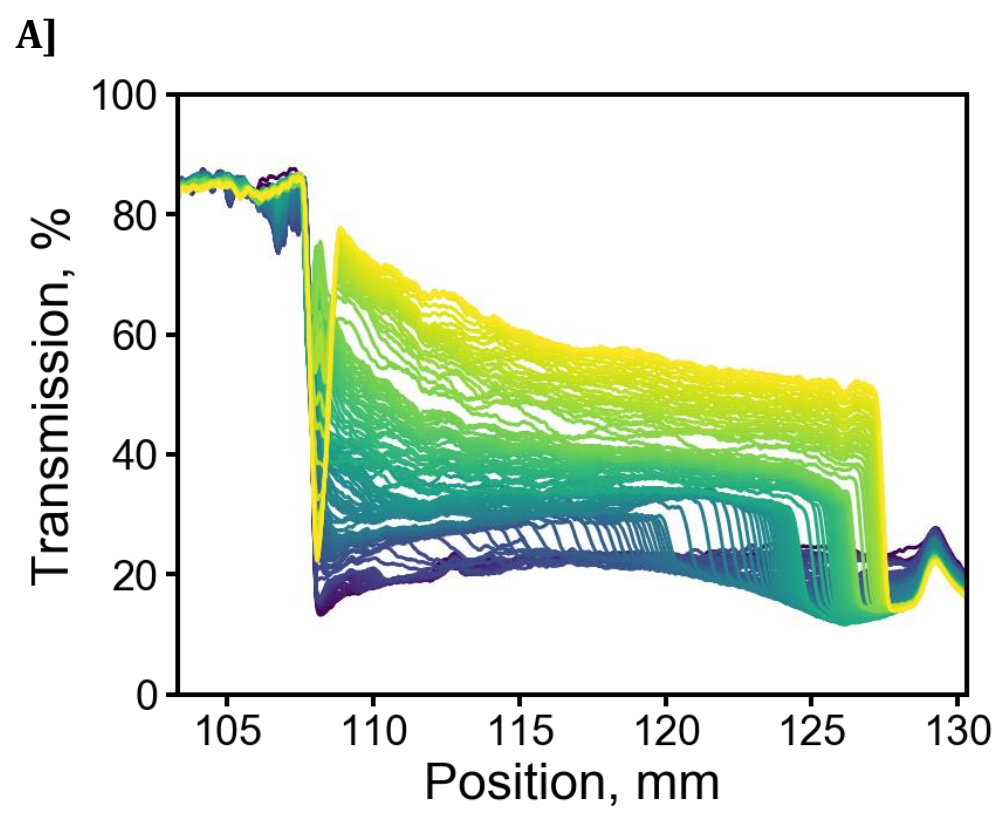

B]

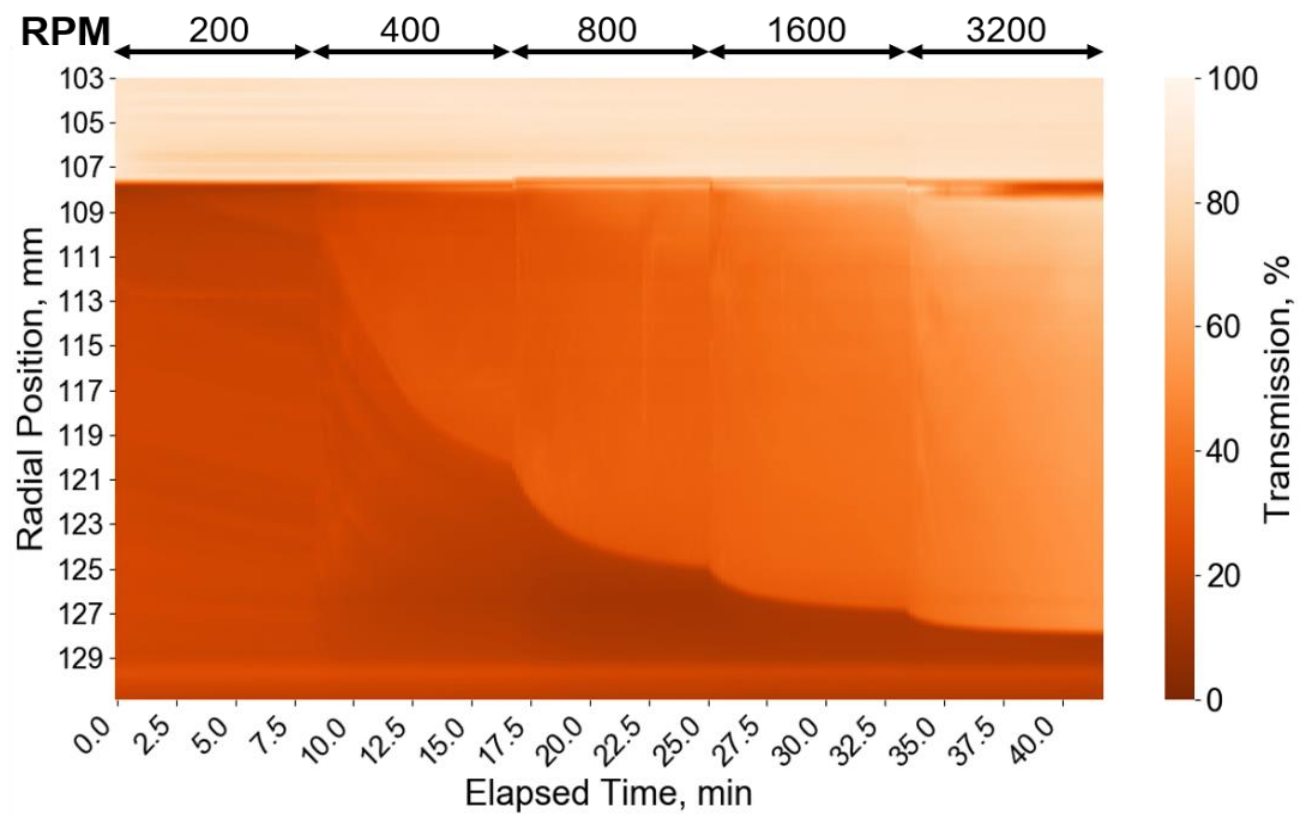

Figure S5: (A) Fingerprints for orange juice measured using analytical centrifuge at constant time interval of 10 seconds, varying speed from $200 \mathrm{RPM}$ to $3200 \mathrm{RPM}$ at $7{ }^{\circ} \mathrm{C}$. (B) Transmittogram for orange juice employing linear color mapping approach. 


\subsection{Color mapping in transmittograms}

Assignment of colors to different transmission values to construct the transmittogram is the key part to construct. Color mapping can be done either linearly, or non-linearly. Additionally, color breaks can be intelligently assigned based on distribution of the underlying transmission values.

\subsubsection{Non-linear and quantile-based color mapping}

Figure S6A shows the transmission profiles for a mixture of silica NPs of four different sizes (sample SM4, see Table S1). The corresponding transmittogram based on linear mapping (Figure S6B) shows at least three sedimentation fronts. With the help of power transformation (Figure S6C), the strata can be better visualized. The visual contrast can further be improved using an automatic quantile-based approach as shown in Figure S6D. This way, the four different sedimentation fronts can be better visualized. 
A]

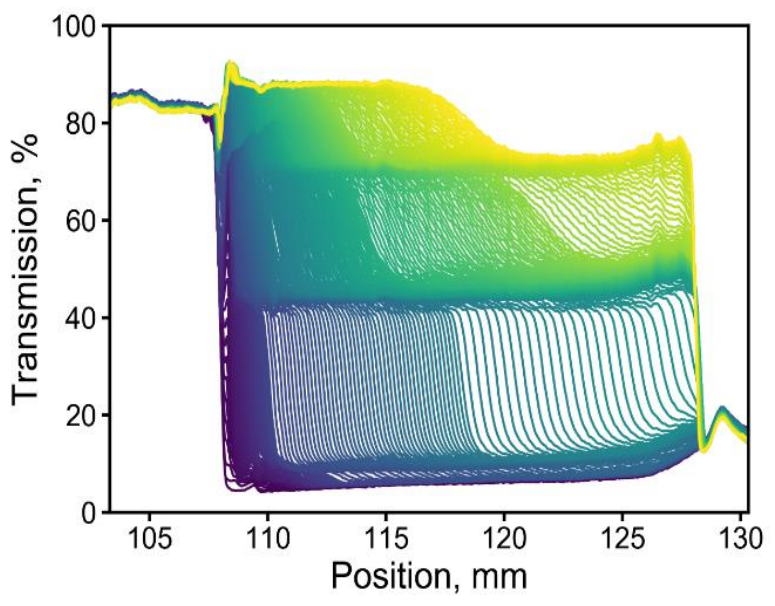

C]

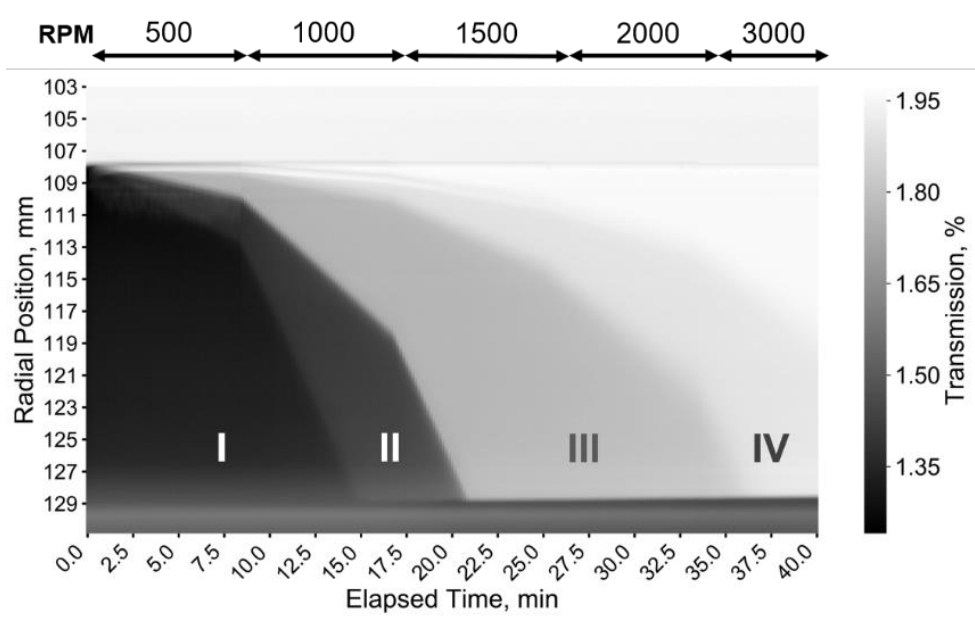

B]

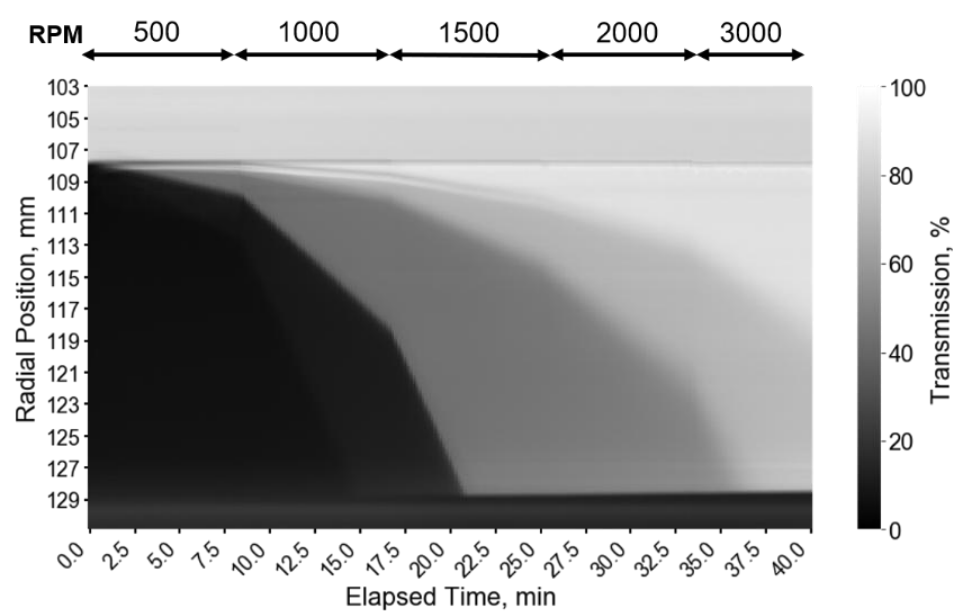

D]

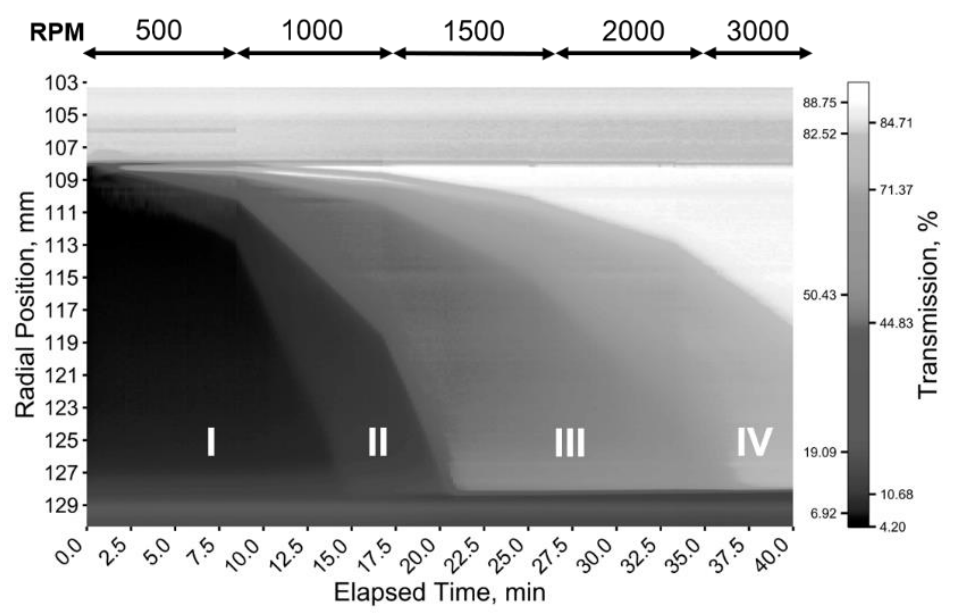

Figure S6: Sedimentation behavior of a mixture of silica NPs of four different sizes (sample SM4). (A) Fingerprints are plotted for 5 wt. \% silica NPs of sizes $150 \mathrm{~nm}, 200 \mathrm{~nm}, 500 \mathrm{~nm}$, and $750 \mathrm{~nm}$ in water mixed at 4:3:2:1 mass ratio. (B) Corresponding transmittogram based on linear color mapping. Here transmission values from $0 \%$ to $100 \%$ are linearly mapped to a monotonically increasing color map (C) Transmittogram using power transformation of the data with power value of 0.15 , followed by color mapping. (D) Transmittogram with quantile-based color mapping for improved visual contrast. Here color breaks are assigned for optimal contrast. 


\subsubsection{Color mapping and underlying data distribution}

The underlying distribution of the transmission values can sometimes be a narrow gaussian distribution. When the transmission values are distributed only over a small range of values, on color mapping effective threshold becomes difficult to be able to distinguish foreground (sedimentation fronts of dispersed phase) from background (solvent phase). This can be observed here in case of a dilute fuel cell ink sample in Figure S7. Gathering any information from transmittogram images becomes difficult in such scenario. Figure S7A shows the distribution of all the transmission values gathered over space and time for a dilute solution

of fuel cell catalyst ink. The sedimentation behavior cannot be easily perceived using transmittograms based on linear mapping (Figure S7B), power transformation (Figure S7C), and quantile break color mapping (Figure S7D). 
A]

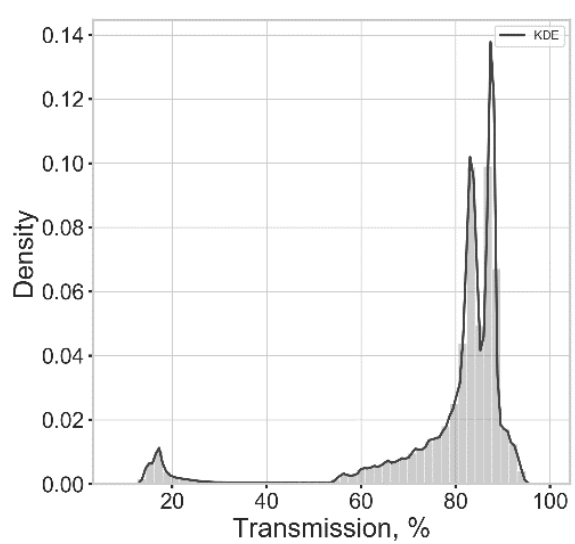

C]

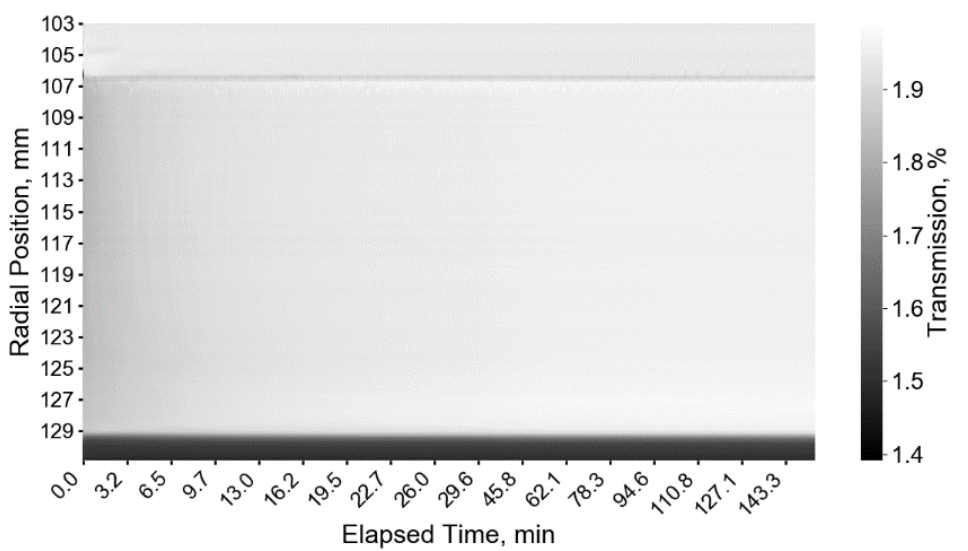

B]

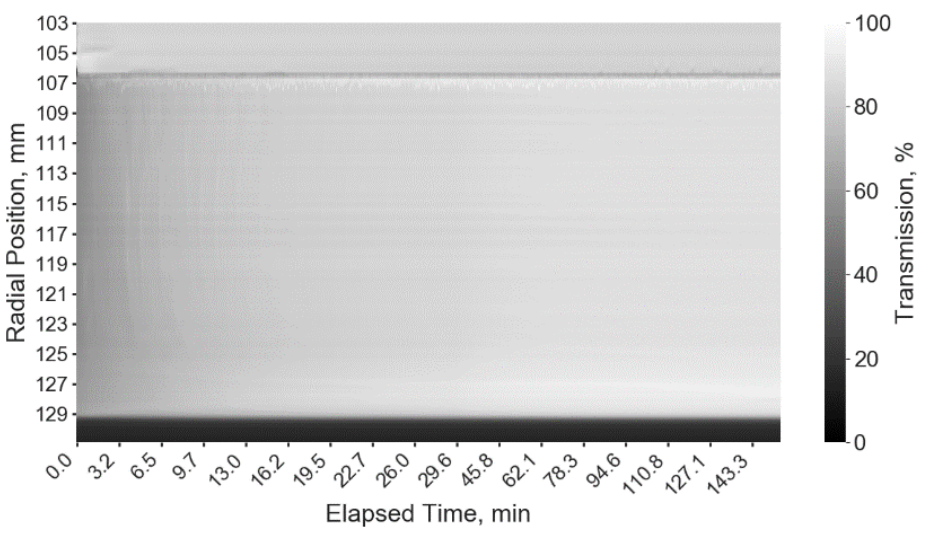

D]

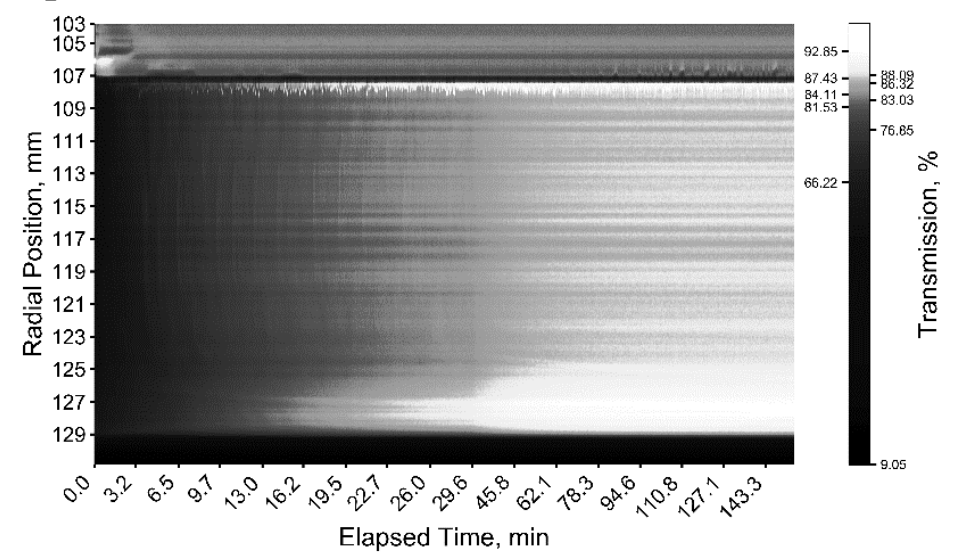

Figure S7: Comparison of different color mapping approaches to construct a transmittogram for a sample which are challenging to be visualized. The sample plotted here is a dilute solution of fuel cell ink. (A) Histogram showing the distribution of transmission values over space and time, along with a kernel density estimated curve. Due to the nature of a tight distribution, it is difficult to effectively threshold at a particular grey value on color assignment, making it hard to discern any sedimentation dynamics. (B) Corresponding transmittogram based on linear mapping. (C) Transmittogram based on power transformation. (D) Transmittogram constructed using the quantile break color mapping approach. Together nonlinear and quantile-based color mapping do not provide added benefit in this case of visualization. 


\subsubsection{Use of perceptual color maps}

Figure S8 shows the transmittogram for catalyst ink FCAT_E20 constructed using a different color mapping (viridis color map). When comparing the transmittogram in Figure S8 with Figure $5 \mathrm{~A}$ (in the main text) one confounded assemblage can be additionally visualized (assemblage $\alpha$ ). This assemblage sediments in $\sim 4 \frac{1}{2}$ hours. This assemblage cannot be distinguished visually in Figure $5 \mathrm{~A}$ as the transmission values in range of $10 \%$ to $30 \%$ are close shades of dark gray. Whereas, in Figure S8, the viridis color map has changing color gradients from dark violet to indigo to blue, green, yellow. This makes visual perception better, leading to enhanced readability of the transmittogram.

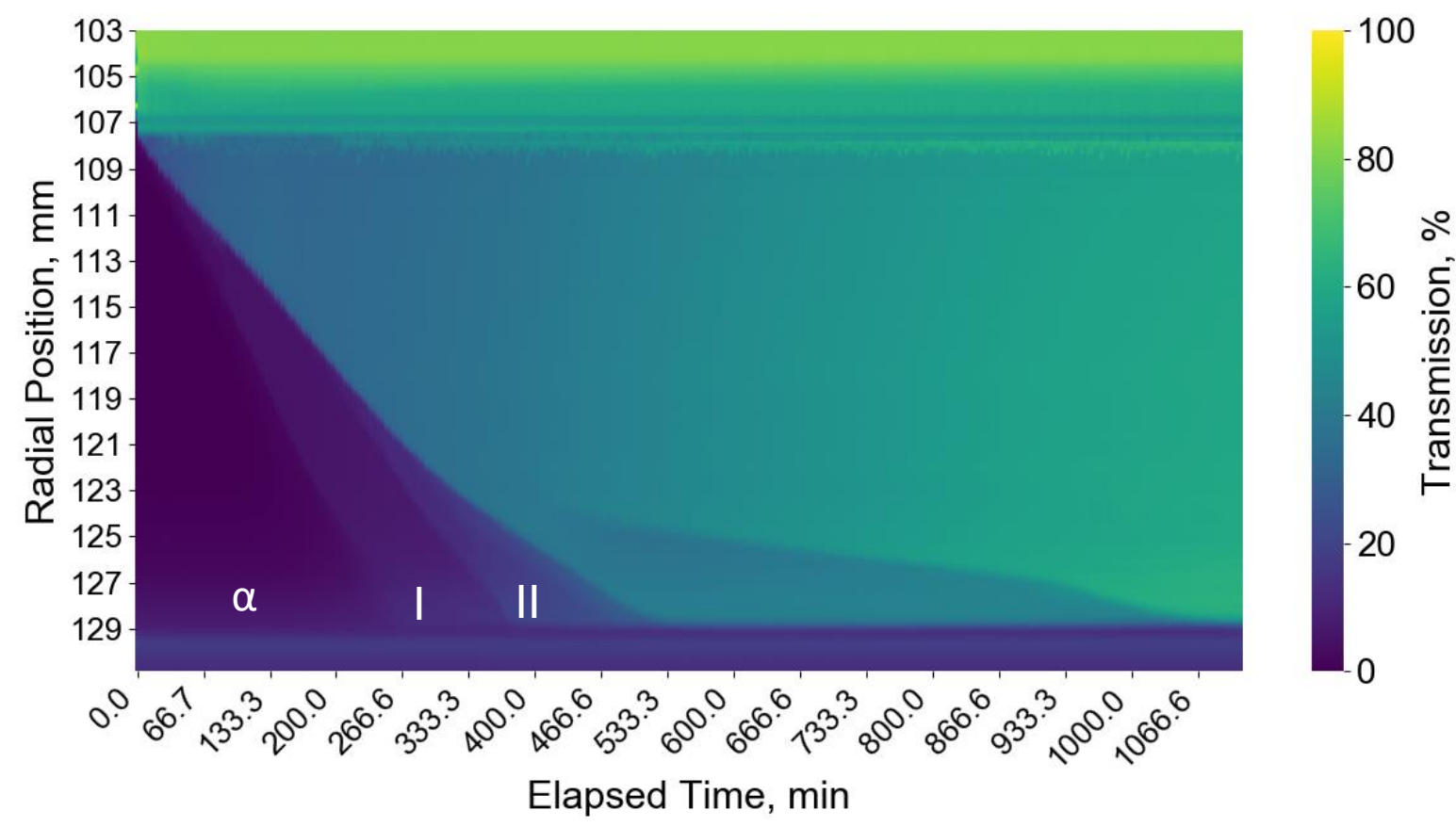

Figure S8: Transmittogram showing sedimentation time-lapse for the catalyst ink FCAT_E20 constructed based on linear mapping of 'viridis' color map. In contrast to using a monotonically increasing colormap, a confounded assemblage $(\alpha)$ can be additionally revealed.

\section{References}

(1) Oliphant, T. E. A Guide to NumPy; Trelgol Publishing USA, 2006; Vol. 1.

(2) McKinney, W.; others. Data Structures for Statistical Computing in Python. In Proceedings of the 9th Python in Science Conference; 2010; Vol. 445, pp 51-56.

(3) Hunter, J. D. Matplotlib: A 2D Graphics Environment. Comput. Sci. Eng. 2007, 9 (3), 90-95. 
